\title{
Assessment of Hearing in Children with Cerebral Palsy
}

\author{
Khaydarova GS, ${ }^{1}$ \\ Madrimova $\mathrm{A}^{2}$
}

Shaykhova Kh.E S ${ }^{3}$

\begin{abstract}
The objective of this study is to identify the prevalence and nature of auditory analyzer pathology in children with different forms of infant cerebral palsy by recording otoacoustic emission and recording short latency brainstem auditory evoked potentials. The subject group consisted of 75 children, $40(53 \%)$ males and $35(47 \%)$ were females, ranging from 3 to 14 years of age with a confirmed diagnosis of cerebral palsy on the basis of a psycho-neurological hospital in Tashkent. (Uzbekistan). The examination of children was carried out by the following methods: clinical examination of patients (general and otorhinolaryngological) and functional research methods of the auditory analyzer. All children underwent acoustic impedancemetry, including tympanometry with determination of the type of tympanogram and acoustic reflex. The data obtained revealed a bilateral lesion of the peripheral part of the auditory analyzer. Beilateral sensorineural hearing loss was detected in 21 - I - II degree, in 12 - III degree and in 15 - IV degree. Marked changes in the main parameters of BAEP were detected in the study of the stem structures of the auditory analyzer in children with cerebral palsy. Thus, disorganization, configuration disturbances of the component composition (I, III, V) were noted. Thus, the study revealed a disorder of auditory function by sound-detecting type and justified the need for hearing correction in these children. Evaluation of auditory function made it possible to determine the level of auditory analyzer injury and localization of the process from the results of a complex, objective examination.
\end{abstract}

Keywords: Cerebral palsy, hearing impairment, otoacoustic emission, brainstem auditory evoked

Department of Otorhinolaryngology Otorhinolaryngology and stomatology, Tashkent Medical Academy, Tashkent, Uzbekistan, E-mail: drdjuraev@mail.ru Phone: +998919920005

Paper submitted on February 02, 2020; and Accepted on December 08, 2020 


\section{INTRODUCTION}

Cerebral palsy is a complex disease of the central nervous system, affecting not only body movement and muscle coordination, but leading also to complications such as hearing impairment, which in turn affects the delay in speech and mental development ${ }^{1-7}$. In children with cerebral palsy, due to the delay of brain development or its pathology of development, there may be a later formation of speech function, these children understand the speech addressed to them, but their own speech is formed at a later date ${ }^{3}$. At the same time in pathogenesis of speech pathology the leading disorder can be damage of auditory analyzer, and disorder of hearing-motor conditional connections leads to insufficiency of motor component of speech in children with cerebral palsy, which further forms delay and underdevelopment of phonematic hearing ${ }^{8-10}$. According to various sources, cerebral palsy develops in 2-3.6 cases per 1000 live births and is the main cause of childhood neurological disability in the world. The prevalence of cerebral palsy in Uzbekistan is 3.7 cases per 1000 newborns (Mutalova Z.D. The structure of diseases of children with disabilities in Uzbekistan under 16 years old (2012-2014) Institute of Health and Medical Statistics). Among premature babies, the incidence of cerebral palsy is $1 \%$. In newborns with a body weight of less than $1500 \mathrm{~g}$, the prevalence of cerebral palsy increases to $5-15 \%$, and with extremely low body weight - up to $25-30 \%{ }^{11-14}$. Thus, the leading and main ones in cerebral palsy are motor disorders, in case of change of muscle tone, coordination disorders, presence of pathological synsikinesis and senergia, involuntary movements, as well as disorders of pose. Motor disorders have an adverse effect on the formation of mental, speech and auditory functions (Alieva Z.S., $2001,2005)^{15}$. A frequent disorder in children with infantile cerebral palsy is speech disorder, the frequency of which according to some authors is between 65 and $85 \%$. Speech disorders can be associated with both deep mental retardation and the deafness bordering deafness. Often these speech disorders are combined ${ }^{15-18}$. In addition to damage to certain brain systems, secondary underdevelopment or later formation of those parts of the cerebral cortex that are extremely important for speech function and most strongly develop already in postnatal ontogenesis (premotor-frontal, dark-temporal regions of the cerebral cortex) plays an essential role in the genesis of speech disorders in these children (Klossovsky B.N.,
1949; Megun G., 1960, Anokhin ILK., 1961) ${ }^{19}$. Since speech is a complex multi-level functional system, correction of speech disorders in children with cerebral palsy is important, along with the study of auditory function. Due to the fact that speech impairment may be due to hearing loss ${ }^{19-25}$.

Four main forms of cerebral palsy are distinguished depending on muscle tone disorders

- $\quad$ spastic

- $\quad$ ataxic

- dyskinetic (subdivided into athetoid and dystonic forms)

- $\quad$ Mixed forms of cerebral palsy.

Cerebral palsy is also classified regarding the affected parts of the body

- Hemiplegia (damage to one half of the body - right or left),

- Diplegia (paralysis that affects both halves of the body, but more of the legs),

- Tetraplegia (damage to the arms and legs).

According to (Semenova K.A., Makhmudova N.A.1979) hearing impairment is noted in $10-40 \%$ of children with cerebral palsy, more often with a hyperkinetic form. When a diagnosis is correct and made in a timely manner, it gives an opportunity, as early as possible, to begin correction of auditory disorders and integration of the child into the speech environment, thus improving the quality of life of the baby ${ }^{26-29}$.

\section{METHODS}

The subject group consisted of 75 children, 40 (53\%) males and $35(47 \%)$ were females, ranging from 3 to 14 years of age. Clinical examination of children began with a thorough history of the child, starting from the moment of birth to the present period. The largest number of children 48 , which amounted to $64 \%$ (Table 1), was with frequent forms of cerebral palsy (spastic diplegia). A significant part of the children's history had a combination of several risk factors that inevitably entail the addition of other factors and thereby sharply increase the likelihood of hearing loss. Such a factor, for example, is birth weight less than $1500 \mathrm{~g}$, which entails hypoxia, the need for

Table 1: The distribution of the examined children in the form of cerebral palsy

$\begin{array}{cccc}\text { Forms of cerebral palsy } & \text { Amount of children } & \text { Total } \\ \text { Spastic diplegia } & \text { M } & \text { D } & 48 \\ \text { Hemiparetic } & 27 & 21 & 12 \\ \text { Atonic-astatic } & 9 & 3 & 8 \\ \text { Hyperkinetic } & 4 & 4 & 5 \\ \text { Bilateral hemiplegia } & 1 & 4 & 2 \\ \text { Total: } & 2 & - & 75\end{array}$


prolonged mechanical ventilation and the use of ototoxic drugs. Thus, it was found that most of the examined children 53 (70.7\%) were born prematurely (from 24 to 36 weeks of gestation), 48 (64\%) of children with low body weight. At $64.5 \%$ (31 people) had burdened obstetric history: a history of abortion had a 15 (31\%) women, the share of abortions and dead born had by $10.4 \%$ and $12.5 \%$, respectively. Analysis and statistical processing of $23(48 \%)$ labor histories of pregnant women with various gestoses. The examination of children was carried out by the following methods: clinical examination of patients (general and otorhinolaryngological) and functional research methods of the auditory analyzer. All children underwent acoustic impedancemetry, including tympanometry with determination of the type of tympanogram and acoustic reflex. When registering tympanograms, $48 \%$ of children received type "A", $31 \%$ - type "B" and $21 \%$ - type "C". In order to differential diagnosis of conductive and sensorineural hearing loss, as well as to study the correlation of data obtained during acoustic impedancemetry depending on the condition of the middle ear, children were recorded caused by otoacoustic emission at the frequency of the distortion product. The stimulus intensity was used in the range from 50 to $70 \mathrm{~dB}$, while the level of two tones was the same or varied by 5-10 dB. Based on measurements of the DPOAE amplitude obtained by presenting tones of different frequencies, a DP gram was automatically constructed a graph of the dependence of the DPOAE amplitude on the tone frequency. For each point of the curve, the background noise level was also calculated. The amplitude of DPOAE exceeded the noise level by at least $3 \mathrm{~dB}$. One of the main objectives of this study was the differential diagnosis of auditory analyser disorders. Currently, the use of acoustic auditory evoked potentials is of great importance to clarify the extent and location of lesions of the auditory analyser. For this purpose, children underwent an electrophysiological study of hearing using the method of recording short latency brainstem auditory evoked potentials - an assessment of the state of the stem structures of the auditory analyzer. When registering the BAEP, broadband clicks, tonal pulses with a rise frequency and a duration of $2 \mathrm{~ms}$ were used as sound stimuli; with a presentation frequency of $11 / \mathrm{sec}, 2,000$ responses accumulated, analysis time - 20 ms; bandwidth - 30-1500 (12 dB per octave).

\section{RESULTS AND DISCUSSION}

The data obtained revealed a bilateral lesion of the peripheral part of the auditory analyzer. Beilateral sensorineural hearing loss was detected in 21 - I - II degree, in 12 - III degree and in 15 - IV degree. Marked changes in the main parameters of BAEP were detected in the study of the stem structures of the auditory analyzer in children with cerebral palsy. Thus, disorganization, configuration disturbances of the component composition (I, III, V) were noted. The smoothness of the components was noted due to a decrease in the amplitude of the response by 2-2.5 times. The analysis of time parameters showed that, with a similar dependence of latency on changes in the intensity of the stimulus in children with cerebral palsy, in contrast to the norm, the time parameters of the main components of the BAEP $(\mathrm{I}, \mathrm{II}, \mathrm{V})$ are significantly delayed: I - by $0.1 \pm 0,6$; III - by $0.2 \pm 0.06$ and by $\mathrm{V}$ - by $0.37 \pm 0.07$ ms. Peak intervals of the main components I - III; I - V; III - V are increased in comparison with the norm in the whole range of reactivity of BAEP. Studies have shown that the most pronounced peak-to-peak I - V intervals are increased by $0.18 \mathrm{~ms}$ compared to normal. In children with cerebral palsy with deep hearing impairment, a narrow dynamic range is observed, on average not exceeding 40-45 ms. An L - shaped dependence of latency on the increase in stimulus intensity was revealed; a significantly higher percentage of impaired volume function is noted. Thus, the study allowed us to identifyimpaired auditory function in the sound-absorbing type in children with cerebral palsy. Assessment of auditory function allowed the results of a comprehensive, objective examination to determine the level of damage to the auditory analyzer and the localization of the process, as well as conduct electro-acoustic rehabilitation of this category of children. As you know, the leading and main in cerebral palsy are motor disorders that have an adverse effect on the formation of mental, speech and auditory functions [26]. A frequent violation in children with cerebral palsy is speech disorder, the frequency of which, according to some authors, ranges from 65 to $85 \%$, while others from 70 to $80 \%$ of children. Speech disorder may be delayed speech development (late formation of the motor component of speech, especially its sound-producing side), dysarthria (pseudobulbar, extrapyramidal, cerebellar, cortical). Speech disorders can be associated with both deep mental retardation and hearing loss bordering deafness. Often, these speech disorders are combined. The study of the stem structures of the auditory analyzer in children with cerebral palsy, having normal thresholds of auditory sensitivity, revealed

\section{Pronounced changes in the main parameters of the} BAEP: disorganization, disturbances in the configuration of the component composition (I, II, V) were noted; smoothing of components was noted due to a decrease in the amplitude of the response by a factor of 2-2.5. Analysis of time parameters showed that, with a similar dependence of latency on changes in stimulus intensity in children with cerebral palsy, in contrast to the norm, the time parameters of the main components of short avoked breain (I, II, V) significantly tightened: I - by $0.1 \pm 0.7$; III by $0.18 \pm 0.05$ and by $V$ - by $0.39 \pm 0.08 \mathrm{~ms}$. Peak intervals of the main components I - III; I - V; III - V are increased in comparison with the norm in the whole range of reactivity of SVP. Studies have shown that the most pronounced peak-to-peak I - V intervals are increased by $0.18 \mathrm{~ms}$ compared to normal. In children with cerebral palsy with deep hearing impairment, a narrow dynamic range is observed, on average not exceeding 40-45 ms. An L shaped dependence of latency on the increase in stimulus intensity was revealed, a significantly higher percentage 
Table 2: The values of BAEP depending on the etiological factor of cerebral palsy.

$\begin{array}{ccccc}\text { Etiological factor } & \text { Number of patients } & \text { Peak Interval I-V } & \text { Amplitude } & \text { \% To I wave ratio } \\ \text { Very low weight } & 9 & 3.93 \pm 0.25 & 0.35 \pm 0.10 & 1.25 \pm 0.66 \\ \text { Asphyxia } & 26 & 4.01 \pm 0.24 & 0.28 \pm 0.09 & 1.02 \pm 0.67 \\ \text { Unknown etiology } & 12 & 4.07 \pm 0.27 & 0.32 \pm 0.12 & 1.14 \pm 0.72 \\ \text { Control group } & 25 & 3.95 \pm 0.18 & 0.37 \pm 0.10 & 1.25 \pm 0.61\end{array}$

of impaired volume function (FCFN) was noted. In a comparative aspect, the signal response in children with cerebral palsy was less mature. The amplitudes of the waves of auditory evoked potentials, as a rule, were smaller in comparison with normal values. In contrast, the peak-to-peak intervals in most cases corresponded to normal values. Table 2 presents the values of BAEP depending on the etiological factor of cerebral palsy

In the history of the mother, we paid great attention to the course of pregnancy and childbirth. The presence of toxicosis in the mother, its course and duration, the use of ototoxic drugs during the pregnancy (aminoglycoside series, "loop" diuretics). Of the 9 children we examined with cerebral palsy, 4 children were born using cesarean section before the 30th week of gestational age, of which three children had birth weight less than $1500 \mathrm{~g} .2$ children born on time had hyperbilirubinemia (bilirubin content of more than $20 \mathrm{mmol} / \mathrm{l}$ ). With hemolytic disease of full-term newborns, there were 3 children. A significant portion of the children have a history of a combination of several risk factors. Some of them inevitably entail the addition of other factors and thereby sharply increase the likelihood of hearing loss. Such a factor, for example, is birth weight less than $1500 \mathrm{~g}$, which entails hypoxia, the need for prolonged mechanical ventilation and the use of ototoxic drugs. In most cases, in children with cerebral palsy, the amplitude of $I$ and $V$ waves and the ratio of $V$ waves to $I$ were less than the average values characteristic of this age. Thus, the obtained values showed a significant difference in the amplitude of the $\mathrm{V}$ wave during cerebral palsy, due to the birth of very low body weight, asphyxiation, and unknown etiology. The amplitude of the $\mathrm{V}$ wave in children born with a very low birth was slightly reduced, but did not significantly differ from the control group. Some children showed poor identification of waves 2 and 4 , sometimes even I waves. Basically, this observation was made in children with cerebral palsy of unknown etiology, who had deep damage to the central nervous system. The results showed that there are no significant differences from the control group in the values of the peak intervals I-V, I-III and III-V in children with cerebral palsy, regardless of its etiology. There were also no significant differences in the interaural difference in the latencies of the $\mathrm{V}$ peaks on both sides of the interaural difference for the interval I-V, I-III and III-V. Compared with the control group, the interval I-V was slightly longer in children with cerebral palsy of unknown etiology. In children with cerebral palsy, against the background of very low body weight, the interval I-V was shortened. As the obtained data show, a significant decrease in the amplitude of the $\mathrm{V}$ wave and, accordingly, the $\mathrm{V} / \mathrm{I}$ ratio is observed in children who survived asphyxia at the beginning of life. There is also a tendency to shortening of the 1st wave, which leads to a relative increase in the $\mathrm{V} / \mathrm{I}$ ratio. Of the 78 children with cerebral palsy, $21(26.1 \%)$ had a significant decrease in the amplitude of the $\mathrm{V}$ wave, and $10(12.5 \%)$ had a significant decrease $\mathrm{V} / \mathrm{I}$ ratio. Absence of waves was noted in 14 cases (17.5\%). Only 10 children with cerebral palsy (12.5\%) had a significant lengthening of the interval I-V, which was often accompanied by a significant decrease in the amplitude of wave V. Deviations in intervals I-III and III-V and ratios III-V / I-III were rare. An increase in intraural difference (6.3\%) was noted in $5(6.3 \%)$ cases. This increase was mainly due to the unilateral shortening of intervals III-V and IV (2 children with very low birth weight) or the unilateral extension of 2 intervals ( 2 children with unknown etiology of cerebral palsy). In total, 48 out of 75 children with cerebral palsy had deviations in auditory evoked potentials, which manifested itself in a significant decrease in the amplitude of the $\mathrm{V}$ wave, a decrease in the $\mathrm{V} / \mathrm{I}$ ratio, an increase in the IV interval, and the absence of I or more response waves. The most often seen deviations were manifested during the registration of the $V$ wave, which appeared by a decrease in its amplitude. In some children with cerebral palsy, more than one deviation from normative indicators was recorded. These children often, although not always, had profound disorders of the central nervous system. The results showed a significant difference between children with cerebral palsy and the control group. The present study showed that the main changes in cerebral palsy are manifested in the form of depression of the signal and a decrease in the amplitude of the $\mathrm{V}$ wave, as a rule. These changes mainly reflect a decrease in the size, or synchronization of neural activity in the auditory part of the brain. These changes may be due to neurological abnormalities or be directly a manifestation of cerebral palsy. Summarizing, we can say that there is a depression of waves, including wave I. A decrease in the amplitude of the I wave is observed, accordingly, the $\mathrm{V} / \mathrm{I}$ ratio increases. As a result, even with a significant decrease in the amplitude of the 5 wave, the $\mathrm{V} / \mathrm{I}$ ratio changes little and remains within the normal range. This can largely explain why a significant decrease in the $\mathrm{V} / \mathrm{I}$ ratio is less often detected compared to a decrease in the amplitude of the 5th wave. This result shows that, compared with the $\mathrm{V} / \mathrm{I}$ ratio, the 5-wave amplitude is more sensitive when registering auditory evoked potentials with a reliable reflection of auditory function in clinical practice. When assessing auditory evoked potentials, it should be taken into account that 
waves 2 and 4 are characterized by great variability and are less reliable than waves I, III, and V. In assessing the configuration of the curve, in some cases precipitation of individual components was noted. The curves with the loss of the first, third, and fifth peaks were not encountered in the analysis. The maximum loss occurred in the second, fourth and sixth components. According to the established opinion and literature data, it is these peaks that are distinguished with the least frequency. However, according to its own regulatory data, the secrecy of these peaks does not differ much from the secrecy of the rest, based on which we can conclude that configurational changes are quite common with spastic diplegia. The technique for recording auditory evoked potential includes mandatory monotonous audiometry, which allows you to get an idea of the state of auditory function. An increase in the auditory threshold above the laboratory norm was noted in 18 (30\%) cases in $34(28.3 \%)$ studies. That is, almost every third patient had a one-sided increase in the auditory threshold. The main trends in the parameters of the short-latency auditory evoked potential were a decrease in the amplitude and an increase in the latency of the components. A significant $(p<0.05)$ increase in latency was noted for the I, II, III, IV and VI components of the potential. An increase in absolute latencies per se cannot be a sign of conduction disturbance at the stem level, especially given how often disturbances in the function of the peripheral part of the analyzer were encountered. However, a significant increase in the inter-peak interval III - V indicates dysfunction of stem afferents at the bridge-mesencephalic level. The detected decrease in the amplitude of the I, II, $V$ components of the potential $(p<0.05)$ is most likely a consequence of the dysfunction of the peripheral unit of the analyzer. A fall in the amplitude $\mathrm{V} / \mathrm{I}$ ratio below 0.75 was observed in $20 \%$, below 0.5 - in $6.7 \%$ of patients in 5 $(4.2 \%)$ studies. The analysis of peak-to-peak intervals indicates the truth of the revealed changes in the conductive ability of stem afferents. The analysis of amplitude relationships allows us to confirm the presence of a nuclear-dysfunctional component in the pathophysiological picture of spastic diplegia, however, the frequency of occurrence does not allow us to see in it the key mechanism of the pathogenesis of stem disorders present in the clinical picture of the disease. The revealed changes in the conductive properties of stem afferents contribute to the picture of the dysfunction of stem structures during spastic diplegia and can have residual demyelination nature. Thus, the study revealed a disorder of auditory function by sound-detecting type and justified the need for hearing aids in these children. Evaluation of auditory function made it possible to determine the level of auditory analyzer injury and localization of the process from the results of a complex, objective examination.

\section{CONCLUSION}

The use of objective methods for the study of hearing in children with cerebral palsy allows you to identify the nature and extent of hearing impairment. The pathogenesis of cerebral palsy combines dysfunction of the nuclear apparatus of the brain stem and impaired conductive properties of stem afferents. The study of hearing at an early age with the help of objective methods helps to timely detect hearing impairment and delineation of deafness and hearing loss from a number of diseases that delay speech development, which is an important link in the chain of rehabilitation measures aimed at correcting difficult - the structure of the defect in children with Cerebral palsy. Our study indicated the need for an early (from the first days of life) and comprehensive objective examination of auditory function in children with cerebral palsy. Early diagnosis of the pathology of the auditory analyzer provides timely correction of auditory speech disorders in children with cerebral palsy.

\section{CONFLICT OF INTEREST}

There are no conflicts of interest in terms of the present study.

\section{REFERENCES}

1. Topolska MM., Hassmann-Poznańska E, Sołowiej E. Assessment of hearing in children with infantile cerebral palsy. Comparison of psychophysical and electrophysical examination. The Polish otolaryngology. 2002;56:467-74.

2. Reid SM. A population-based study and systematic review of hearing loss in children with cerebral palsym, Developmental Medicine and Child Neurology. 2011;53:1038-45.

3. Hadden KL. A comparison of observers' and self-report pain ratings for children with cerebral palsy, Journal of Developmental \& Behavioral Pediatrics. 2015;36:14-23.

4. Bhatia M, Joseph B. Rehabilitation of cerebral palsy in a developing country: the need for comprehensive assessment in Pediatric rehabilitation. 2000;4:83-6.

5. Steven RA. Cochlear implantation in children with cerebral palsy //International journal of pediatric otorhinolaryngology. 2011;75:1427-30.

6. Kennes J. Health status of school-aged children with cerebral palsy: information from a population-based sample. Developmental medicine and child neurology. 2002;4:240-7.

7. Morris C. Reliability of the manual ability classification system for children with cerebral palsy developmental medicine and child neurology. 2006;48:950-3.

8. Elsayed RM. Sleep assessment of children with cerebral palsy: Using validated sleep questionnaire, Annals of Indian Academy of Neurology. 2013;16:62-4.

9. Morris C. Do the abilities of children with cerebral palsy explain their activities and participation Developmental medicine and child neurology. 2006;48:954-61.

10. Aneja S. Evaluation of a child with cerebral palsy The Indian Journal of Pediatrics. 2004;7:627-34.

11. Rosenbaum P. A report: the definition and classification of cerebral palsy.Dev Med Child Neurol. 2007;109:8-14.

12. Pharoah POD. Epidemiology of cerebral palsy in England and Scotland, Archives of Disease in Childhood-Fetal and Neonatal Edition. 1998;79:F21-F25. 
13. Dickinson H. Assessment of data quality in a multi-centre cross-sectional study of participation and quality of life of children with cerebral palsy BMC public health. 2006;6:2734.

14. Surman G. Children with cerebral palsy: severity and trends over time Paediatric and perinatal epidemiology. 2009;23:513-21.

15. Ashwal S. Practice parameter: diagnostic assessment of the child with cerebral palsy: report of the Quality Standards Subcommittee of the American Academy of Neurology and the Practice Committee of the Child Neurology Society Neurology. 2004;62:851-63.

16. Bacciu A. Cochlear implantation in children with cerebral palsy. A preliminary report. International journal of pediatric otorhinolaryngology. 2009;73:717-21.

17. Arnaud C. Parent-reported quality of life of children with cerebral palsy in Europe Pediatrics. 2008;121:54-64.

18. Sigurdardottir S. Cognitive profile in young Icelandic children with cerebral palsy Developmental Medicine \& Child Neurology. 2008;50:357-362.

19. Morris C. Who best to make the assessment Professionals' and families' classifications of gross motor function in cerebral palsy are highly consistent Archives of disease in childhood. 2006;8:675-9.

20. Yin Foo R, Guppy M, Johnston LM. Intelligence assessments for children with cerebral palsy: a systematic review. Developmental Medicine \& Child Neurology. 2013;55:911-18.

21. Ansel BM, Kent RD. Acoustic-phonetic contrasts and intelligibility in the dysarthria associated with mixed cerebral palsy. Journal of Speech, Language, and Hearing Research. 1992;35:296-308.

22. Sankar C, Mundkur N. Cerebral palsy-definition, classification, etiology and early diagnosis. The Indian Journal of Pediatrics. 2005;72:865-8.

23. Sigurdardottir S, Vik T. Speech, expressive language, and verbal cognition of preschool children with cerebral palsy in Iceland. Developmental Medicine \& Child Neurology. 2011;53:74-80.

24. Dobhal M. Health-related quality of life in children with cerebral palsy and their families Indian pediatrics. 2014;51:3857.

25. Card R, Dodd B. The phonological awareness abilities of children with cerebral palsy who do not speak. Augmentative and Alternative Communication. 2006;22:49-159.

26. Varni JW. Health-related quality of life of children and adolescents with cerebral palsy: hearing the voices of the children. Developmental Medicine and Child Neurology. 2005;47:592-7.

27. Pirila S. Language and motor speech skills in children with cerebral palsy. Journal of communication disorders. 2007; $40: 116-128$

28. Cans C, De-la-Cruz J, Mermet MA. Epidemiology of cerebral palsy. Paediatrics and child health. 2008;18:393-8.

29. Sigurdardottir S. Behavioural and emotional symptoms of preschool children with cerebral palsy: a populationbased study. Developmental Medicine \& Child Neurology. 2010;52:1056-61. 